$\overline{\mathrm{AEET}}$

ASOCIACIÓN ESPAÑOLA DE ECOLOGÍA TERRESTRE

\title{
Competencia entre Zea mays y Sorghum halepense por agua edáfica y su incidencia en el intercambio gaseoso foliar
}

\author{
H. A. Acciaresi ${ }^{1,2, \star}$, M. S. Zuluaga ${ }^{1}$, M. E. Yanniccari ${ }^{3}$, J. J. Guiamet ${ }^{2,3}$ \\ (1) Facultad de Ciencias Agrarias y Forestales, Universidad Nacional de La Plata, C.C. 31 (1900) La Plata, Argentina. \\ (2) Comisión Investigaciones Científicas de la Pcia de Buenos Aires. Argentina. \\ (3) Instituto de Fisiología Vegetal (UNLP - CONICET), C.C. 327 (1900) La Plata, Argentina
}

*Autor de correpondencia: H.A. Acciaresi [acciaresi@agro.unlp.edu.ar]

> Recibido el 1 de febrero de 2011, aceptado el 22 de agosto de 2012

\begin{abstract}
Acciaresi, H., Zuluaga, M., Yanniccari, M., Guiamet, J. (2012). Competencia entre Zea mays y Sorghum halepense por agua edáfica y su incidencia en el intercambio gaseoso foliar. Ecosistemas 21(3):103-109. Doi.: 10.6778/ECOS.2012.21-3.15

La alta estabilidad productiva en ambientes con restricciones hídricas se ha vinculado a la habilidad de conservación del agua por parte de los cultivos. Sin embargo, diferentes especies de malezas, bajo competencia hídrica realizan un uso ineficiente de la misma. Con el objetivo de determinar la estrategia de consumo de agua que poseen Zea mays y Sorghum halepense bajo dos situaciones de disponibilidad hídrica edáfica y trabajando

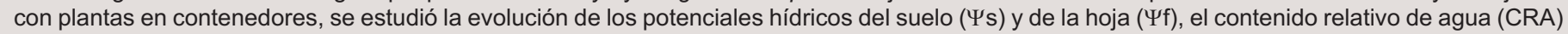
y el intercambio gaseoso foliar durante el período crítico de competencia del cultivo. Además, se calculó la eficiencia en el uso del agua, la complementariedad de recursos por medio de la productividad relativa total (RYT) y la habilidad competitiva a través del índice de agresividad (AGR). Se observó que S.halepense realizó una absorción continua de agua, alcanzando un menor $\Psi$ f respecto de los híbridos de Z.mays. S.halepense mantuvo el CRA por encima del $90 \%$, alcanzado el $70 \%$ sólo en competencia con baja disponibilidad hídrica. En Z.mays, el CRA disminuyó a valores de $70 \%$ en ambos niveles hídricos estudiados, manteniendo S.halepense un activo intercambio gaseoso foliar. Los híbridos de Z.mays presentaron una menor habilidad competitiva que S. halepense para ambos niveles de competencia debido a la estrategia conservadora de la humedad que mostró el cultivo. El uso del agua realizado por la maleza podría ser una de las causas de la mayor agresividad de S.halepense bajo las condiciones estudio.
\end{abstract}

Palabras clave: potencial agua, contenido relativo de agua, RYT, agresividad.

Acciaresi, H., Zuluaga, M., Yanniccari, M., Guiamet, J. (2012). Zea mays and Sorghum halepense water competition and their impact on leaf gas exchange. Ecosistemas 21(3):103-109. Doi.: 10.6778/ECOS.2012.21-3.15

In an environment with water restrictions, the high stability of the production has been linked to the water conservation ability of the crops. However, under water competition, different weed species show inefficient water use. In order to determine the strategy of water consumption of Zea mays and Sorghum halepense under two soil water availability conditions, soil ( $\Psi$ s) and leaf water potential ( $\Psi \mathrm{f})$ trends, relative water content (RWC) and leaf gas exchange were measured during the critical period of crop competition in pot experiments. In addition, efficiency of water use, relative yield total (RYT) and aggressivity index (AGR) of both components were calculated. S. halepense shown a continuous absorption of water, reaching a lower $\Psi f$ than those observed in Z.mays hybrids. S. halepense maintained the RWC above $90 \%$, reached $70 \%$ only in competition with low water availability. In Z.mays, the RWC declined to values of $70 \%$ for both water levels studied. S. halepense showed an active leaf gas exchange. Z.mays hybrids have had a less competitive ability that $S$. halepense for both competition levels due to the conservative water strategy of the crop. Water use by the weed could be a cause of the increased aggressiveness of S.halepense under study conditions.

Key words: water potential, relative water content, RYT, aggressivity.

\section{Introducción}

El agua es uno de los principales recursos limitantes del crecimiento y producción de los componentes vegetales de los agroecosistemas (Bohnert y Bressan, 2001). Las malezas compiten con los cultivos por recursos edáficos limitantes y consecuentemente reducen la cantidad de agua disponible para los mismos (Patterson, 1995). Por esta razón, la competencia por agua en un sistema cultivo-maleza ha sido definida como un aumento del estrés hídrico del cultivo provocado por el consumo de agua de la maleza (Thomas y Allison, 1975). Sin embargo, es necesario que los estudios de competencia por agua aborden la interacción de dos sistemas dinámicos, uno de ellos comprendido por el sistema suelo-plantaatmósfera y el otro por la interacción maleza-cultivo, en vez de sólo considerar a la competencia como una carencia temporal de agua (Rajcan y Swanton, 2001).

Distintos estudios han establecido que, dentro de los valores de contenido relativo de agua (CRA) registrados en los diferentes agroecosistemas y ante una lenta disminución del contenido de humedad edáfica, el cierre estomático conduce a una menor disponibilidad de $\mathrm{CO}_{2}$, aparejando una declinación de la tasa fotosintética (Cornic y Fresneau, 2002). No obstante, se ha observado que di- 
ferentes especies de malezas realizan un uso no eficiente del agua en situaciones de competencia hídrica, realizando un "consumo de lujo" (Stuart et al., 1985; Patterson, 1995). Esta respuesta de las malezas frente a la competencia por agua edáfica se relacionó con el comportamiento de los estomas, que resultan ser menos sensibles a la disminución del potencial foliar respecto a los estomas de los cultivos con los cuales compiten (Acciaresi y Guiamet, 2010).

En el mejoramiento de cultivos, una estrategia adecuada en ambientes con restricciones hídricas está vinculada a la selección de materiales con características asociadas a una alta eficiencia en el uso del agua (Turner, 2001). Esto puede brindarle al cultivo una ventaja competitiva si la maleza presenta un "consumo de lujo" (sostenido uso del agua) durante el período de competencia (Patterson, 1995).

El objetivo del trabajo fue establecer los cambios en el intercambio gaseoso que presentan dos híbridos simples de Zea mays y una población de Sorghum halepense frente a la variación de la disponibilidad hídrica edáfica, a fin de determinar la estrategia en el consumo de agua que poseen ambas especies en competencia hídrica y el efecto que esa estrategia tiene en la habilidad competitiva del cultivo y la maleza.

\section{Materiales y Métodos}

Los estudios se llevaron a cabo en el Instituto de Fisiología Vegetal (Facultad Ciencias Agrarias y Forestales (UNLP), La Plata $\left(34^{\circ} \mathrm{S}, 58^{\circ} \mathrm{W}\right.$, Argentina) durante las temporadas de crecimiento comprendidas entre los meses de octubre a enero de los años $2003 / 2004$ y $2005 / 2006$. Los ensayos se realizaron en contenedores plásticos de $30 \mathrm{dm}^{3}$ de capacidad colocados al aire libre durante todo el período de estudio.

\section{Material vegetal y condiciones de crecimiento}

Se emplearon rizomas de S.halepense recolectados de infestaciones naturales de La Plata e híbridos simples de Z.mays Ax 888 y Ax 840. Se utilizaron contenedores de $30 \mathrm{dm}^{3}$, empleando como sustrato una mezcla de tierra negra tamizada, turba y arena en una proporción de 0.4:0.25:0.35. Se llevó a cabo una fertilización de base equivalente a $8 \mathrm{~g} \mathrm{~m}^{-2}$ de urea y a $10 \mathrm{~g} \mathrm{~m}^{-2}$ de superfosfato triple de calcio para evitar deficiencias de nitrógeno y fósforo. La densidad fue de una planta de cada especie por contenedor. Se mantuvo el estado hídrico del suelo a $-0.03 \mathrm{MPa}$ (capacidad de campo (CC)) hasta los 21 días desde la emergencia, momento en el que se comenzó con los tratamientos de disponibilidad hídrica a los efectos de generar dos niveles de competencia entre ambas especies. Los tratamientos fueron disponibilidad hídrica baja (B, $75 \%$ CC, correspondiendo a un potencial de $-0.04 \mathrm{MPa}(23.5 \%$ $25 \%$ de humedad $\mathrm{P} / \mathrm{P})$ ) y muy baja (MB, con suspensión del riego durante el período de evaluación). Monoculturas de S.halepense y Z.mays se utilizaron como tratamientos control (2 plantas por contenedor). El período de evaluación (24 días) se correspondió con el período crítico de competencia de malezas en Z.mays, desde la $4^{\text {ta }}$ hasta $7^{\text {ma }}$ hoja expandida.

Las mediciones que se realizaron periódicamente fueron: potencial hídrico edáfico $\left(\psi_{\mathrm{s}}, \mathrm{MPa}\right)$ y foliar $\left(\psi_{\mathrm{f}}, \mathrm{Mpa}\right)$, cuantificación del intercambio gaseoso (fotosíntesis $\left(A, \mu \mathrm{mol} \mathrm{CO}_{2} \mathrm{~m}^{-2} \mathrm{~s}^{-1}\right)$, conductancia estomática $\left(g_{s}, \mathrm{mmol} \mathrm{H}_{2} \mathrm{O} \mathrm{m}^{-} \mathrm{s}^{-1}\right)$ y tasa transpiratoria $(E$ $\left.m m o l ~ H_{2} \mathrm{O} \mathrm{m}^{-2} \mathrm{~s}^{-1}\right)$ ) y contenido relativo de agua (CRA, \%) de ambas especies en los diferentes tratamientos estudiados. Las mediciones de $\psi_{\mathrm{f}}$ y CRA fueron realizadas entre 7:30 hs y 8:30 hs.

Se calculó la producción relativa total (RYT, de Wit y van den Bergh, 1965) a fin de cuantificar la posible complementariedad en el uso de recursos de los tratamientos evaluados. La expresión general teniendo para una proporción 1:1 de cada especie fue:

$$
R Y T=\left(Y_{\mathrm{ij}} / Y_{\mathrm{ii}}\right)+\left(\mathrm{Y}_{\mathrm{ji}} / \mathrm{Y}_{\mathrm{jj}}\right)
$$

Donde $Y_{i j}$ y $Y_{j i}$ son las producciones de biomasa aérea por planta de las especies $i$ y $j$ cuando crecen en mezcla, en tanto $Y_{i i} y$ $Y_{j j}$ son las producciones de biomasa aérea por planta de las especies $i$ y $j$ cuando crecen en monocultura. La habilidad competitiva se cuantificó por medio del índice de agresividad (AGR, Mc Gilchrist y Trenbath, 1971), modificado por Satorre y Snaydon (1992) para experimentos aditivos que incluye el valor del RYT en su cálculo. La expresión utilizada fue:

$$
A G R=\left(Y_{\mathrm{ij}} / Y_{\mathrm{ii}}\right)-\left(\mathrm{Y}_{\mathrm{ji}} / Y_{\mathrm{jj}}\right) / R Y T
$$

Donde cada término fue definido para la ecuación (1).

\section{Análisis estadísticos}

Se utilizó un diseño experimental aditivo de bloques completos al azar con cinco repeticiones, siendo cada contenedor una unidad experimental. Para cada variable, se empleó un análisis de varianza multivariado (genotipos $x$ disponibilidad de agua $x$ DDE) utilizando el error estándar de la media (EEM) para analizar los efectos de las especies y tratamientos. Respecto a los datos de complementariedad de recursos (RYT) y la habilidad competitiva (AGR) se llevó a cabo un análisis de la varianza sobre los tratamientos evaluados. Se realizó una transformación de la raíz cuadrada con los datos de ambos índices y de EUA a fin de homogeneizar la varianza del error. Para la realización de los diferentes análisis se utilizó el programa estadístico Statgraphics Plus 5.1.

\section{Resultados}

El análisis de varianza realizado sobre los datos obtenidos para ambos años de estudio, indicó que no hubo un efecto significativo $(p=0.477)$ debido a las dos estaciones de crecimiento analizadas. Las interacciones año*disponibilidad hídrica, año*especie, las interacciones triples y la interacción cuádruple resultaron no significativas $(p>0.05)$, no registrándose cambios de comportamiento de los tratamientos analizados según los años de estudio. De este modo, se promediaron los datos de ambas estaciones de crecimiento.

\section{Potencial hídrico del suelo y foliar}

Se observó que a lo largo del período de evaluación no se registraron diferencias mínimas significativas en los valores de $\psi_{\mathrm{s}}$ entre las monoculturas regadas. El $\psi_{\mathrm{s}}$ en baja disponibilidad hídrica fue significativamente más bajo $(p<0.05)$ que el $\psi_{\mathrm{s}}$ del control a partir de los 9 días de tratamiento (Fig. 1). A muy baja disponibilidad hídrica se observa que el $\psi_{\mathrm{s}}$ disminuyó significativamente $(p<0.01)$ respecto de aquel alcanzado con baja disponibilidad hídrica a partir del cuarto día (24 DDE) de establecido el período de evaluación (Fig. 1). El efecto de la baja disponibilidad hídrica sobre $\psi_{\mathrm{f}}$ de S.halepense produjo una merma significativa $(p<0.05)$ a partir de los 15 días de iniciada la restricción hídrica (Fig. 2a). En tanto, frente a una muy baja disponibilidad hídrica, la reducción $(p<0.05)$ respecto del control se produjo a los 10 días de iniciada la restricción (Fig. $2 a)$. No hubo una merma diferencial del $\psi_{f}$ de S.halepense para cada uno de los híbridos con los que la maleza compitió en presencia de ambos niveles de deficiencia hídrica (Fig. 2a). Respecto de la evolución del $\psi_{\mathrm{f}}$ en Z.mays, la disponibilidad hídrica y la competencia produjeron una merma significativa del $\psi_{\mathrm{f}}$ a los cinco días de iniciadas las evaluaciones (Fig. 2b). El $\psi_{\mathrm{f}}$ disminuyó hasta los 10 días de iniciadas las evaluaciones (31 DDE) para luego estabilizarse sin cambios significativos en los tratamientos ensayados.

\section{Contenido relativo de agua}

La disminución del CRA en S.halepense a lo largo del período de evaluación fue menor $(p<0.05)$ que la observada en Z.mays, siendo los valores de CRA obtenidos en ambos híbridos menores a aquellos registrados en la maleza, para cada uno de los tratamientos analizados (Fig. 3a y Fig. 3b). El efecto de la muy baja disponibilidad hídrica sobre la merma del CRA en S. halepense fue 

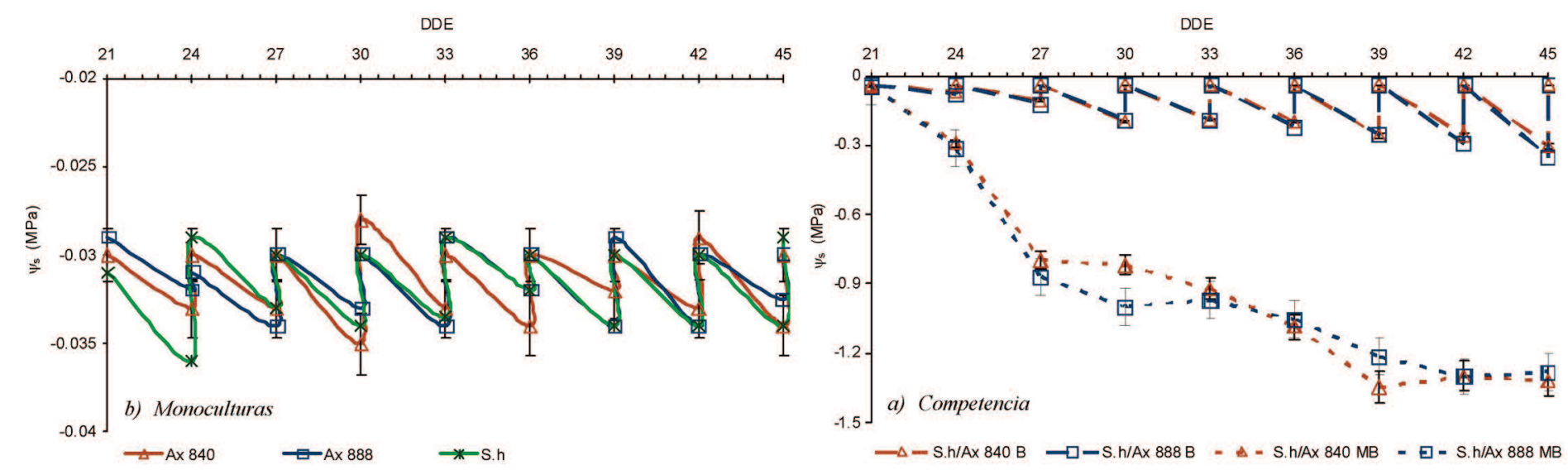

Figura 1. Evolución del potencial hídrico del suelo ( $\left.\psi_{s}, M P a\right)$ a partir del establecimiento de los tratamientos a los 21 días desde la emergencia (DDE) en: a) Monoculturas de Z.mays (híbridos Ax 840 y Ax 888) y S. halepense (S.h); b) Competencia entre S. halepense y Z.mays creciendo con baja disponibilidad hídrica (S.h/Ax 840 B y S.h/Ax888 B) y S.halepense y Z.mays creciendo con muy baja disponibilidad hídrica (S.h/Ax 840 MB y S.h/Ax888 MB). Datos promedios para ambos años de estudio. La barra indica el error estándar (es). ( $n=5)$.
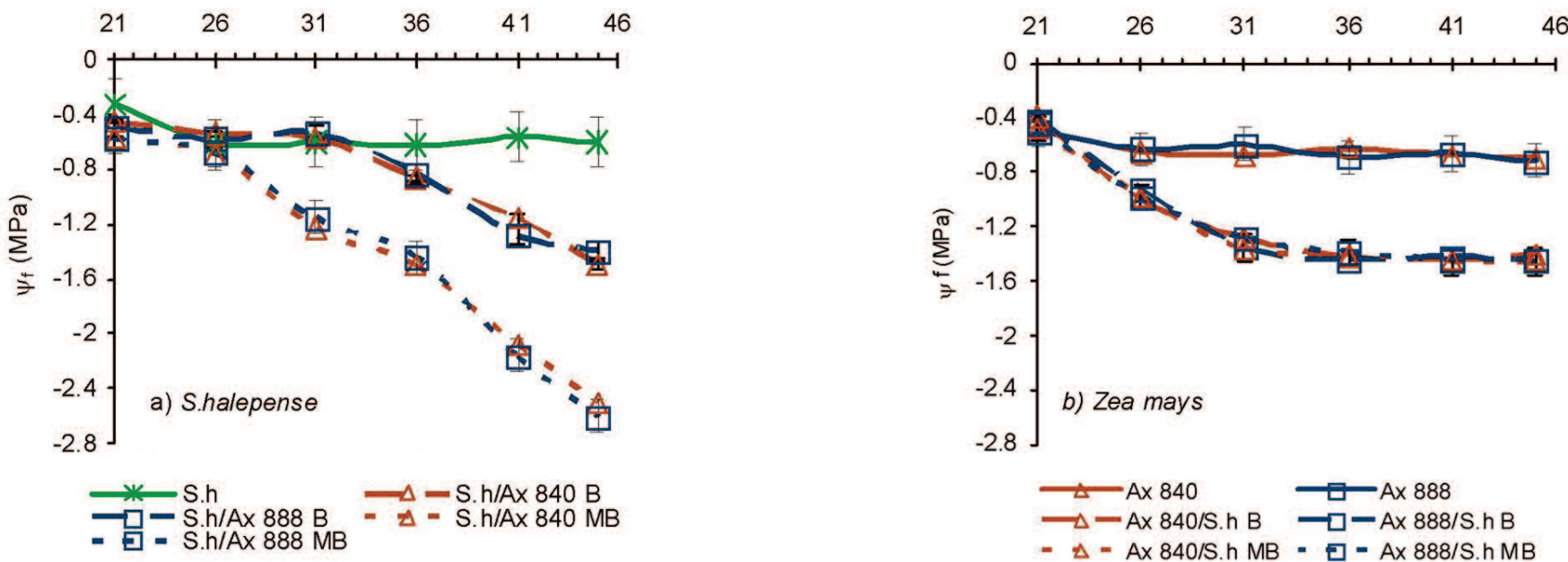

Figura 2. Evolución del potencial hídrico foliar $\left(\psi_{f}, \mathrm{MPa}\right)$ a partir del establecimiento de los tratamientos 21 días desde la emergencia (DDE) en: a) S.halepense (S.h), creciendo con baja disponibilidad hídrica con los híbridos Ax 840 (S.h/Ax840 B) y Ax 888 (S.h/Ax888 B) y con muy baja disponibilidad hídrica (S.h/Ax 840 MB y S.h/Ax888 MB); b) en Z.mays (control, Ax 840 y Ax 888), creciendo con baja disponibilidad hídrica (Ax840/S.h B, Ax888/S.h B) y con muy baja disponibilidad hídrica (Ax840/S. h MB, Ax888/S.h MB). Datos promedios para ambos años de estudio. La barra indica el error estándar (es). ( $n=5)$.
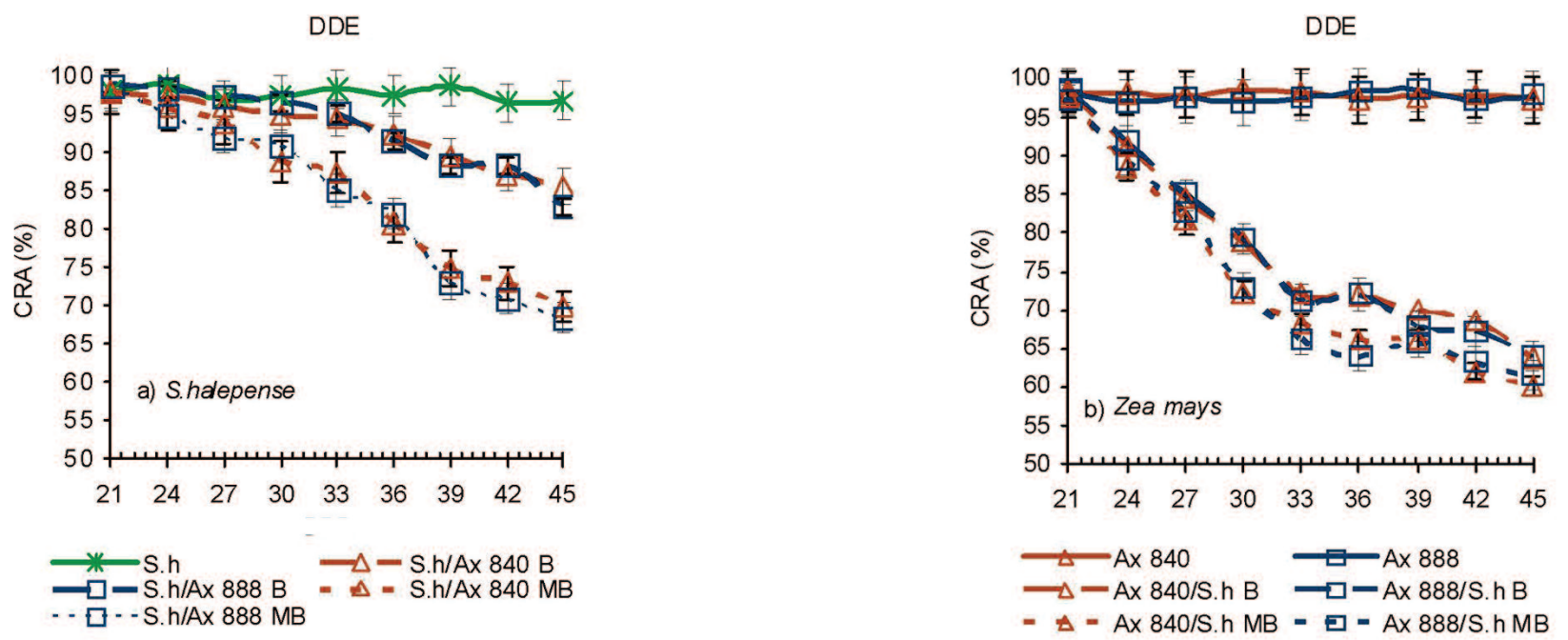

Figura 3. Evolución del contenido relativo de agua (CRA, \%) a partir del establecimiento de los tratamientos 21 días desde la emergencia (DDE) en: a) S. halepense (S.h), creciendo con baja disponibilidad hídrica con los híbridos Ax 840 (S.h/Ax840 B) y Ax 888 (S.h/Ax888 B) y con muy baja disponibilidad hídrica (S.h/Ax 840 MB y S.h/Ax888 MB); b) en Z.mays (control, Ax 840 y Ax 888), creciendo con baja disponibilidad hídrica (Ax840/S.h B, Ax888/S.h B) y con muy baja disponibilidad hídrica (Ax840/S.h MB, Ax888/S.h MB). Datos promedios para ambos años de estudio. La barra indica el error estándar (es). $(n=5)$. 
mayor $(p<0.01)$ que el observado para los otros dos tratamientos a partir de los 12 días de iniciado el período de evaluación. No obstante, recién a los 18 días de iniciado el período de evaluación se obtuvieron valores por debajo del $80 \%$ en S.halepense (Fig. 3a). Sobre el final del período de estudio, la disminución observada en el CRA respecto al control fue de $12.8 \%$ y $30.8 \%$ en los tratamientos de baja y muy baja disponibilidad hídrica, respectivamente (promedio entre híbridos para los dos niveles de competencia) (Fig. 3a).

Se observó una disminución del 15\% en el CRA de Z.mays en la primera semana de evaluación, en tanto en la segunda semana, la merma fue de $29.3 \%$ (promedio de todos los tratamientos). Al final del período de evaluación, la merma promedio fue del $36 \%$ respecto al control de ambos híbridos (Fig. 3b).

\section{Intercambio gaseoso}

Tasa fotosintética, Conductancia estomática al vapor de agua $\left(g_{s}\right)$ y Tasa transpiratoria $(E)$

La evolución de la tasa fotosintética a lo largo del período de estudio mostró una menor variación en S.halepense que en los híbridos de Z.mays (Fig. 4a y Fig. 4b). La merma de la fotosíntesis
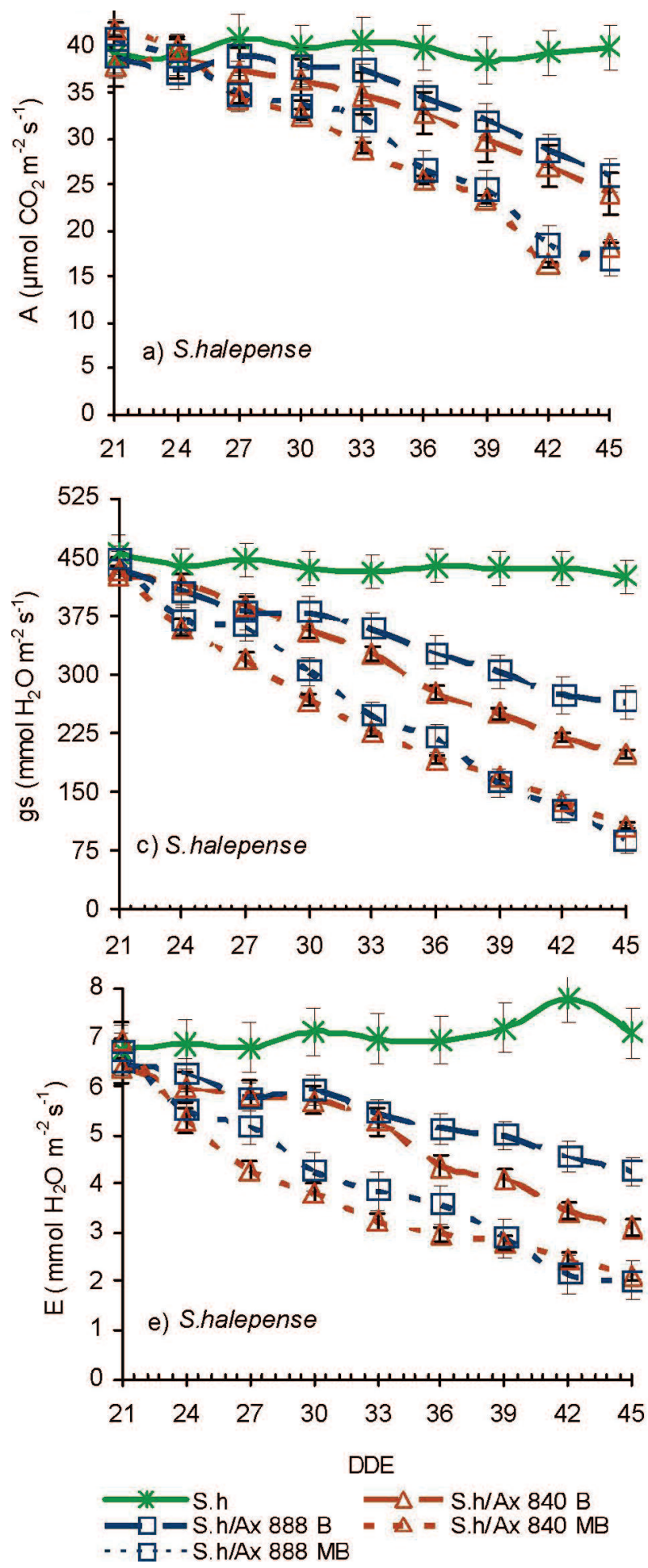
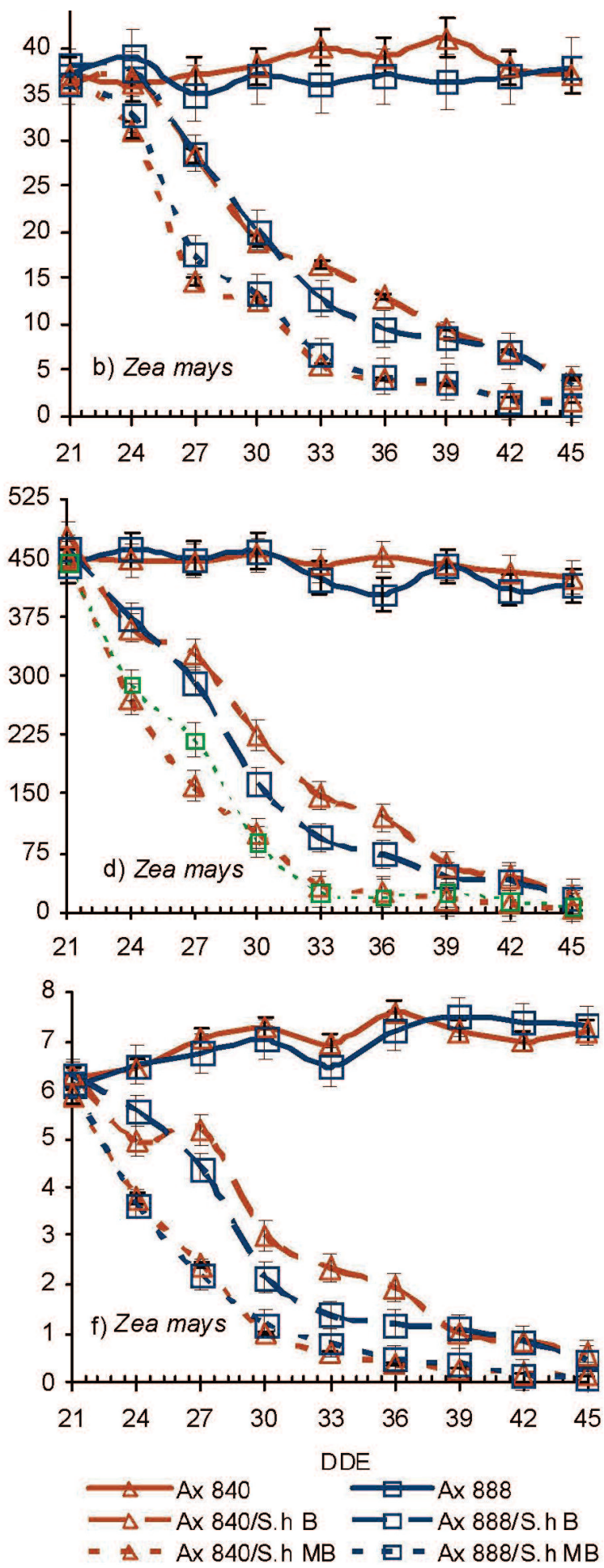

Figura 4. Evolución de la tasa fotosintética $(a, b)(A, \mu m o l m-2 s-1)$, conductancia estomática (c, d) (gs, mmol H2O m-2 s-1) y de la tasa transpiratoria (e, f) (E, mmol m-2 s-1) a partir del establecimiento de los tratamientos 21 días desde la emergencia (DDE) en S.halepense (S.h), creciendo con baja disponibilidad hídrica con los híbridos Ax 840 (S.h/Ax840 B) y Ax 888 (S.h/Ax888 B) y con muy baja disponibilidad hídrica (S.h/Ax 840 MB y S.h/Ax888 MB) y en Z.mays (control, Ax 840 y Ax 888) y creciendo con baja disponibilidad hídrica (Ax840/S.h B, Ax888/S.h B) y con muy baja disponibilidad hídrica (Ax840/S.h MB, Ax888/S.h MB). Datos promedios para ambos años de estudio. La barra indica el error estándar (es). ( $n=5)$. 
de la maleza creciendo con el híbrido Ax 840 con baja disponibilidad hídrica, se diferenció del control $(p<0.05)$ a los 9 días de establecido el período de evaluación. En tanto, con el híbrido Ax 888, la disminución fue significativa $(p<0.05)$ recién a los 15 días de establecida la competencia (Fig. 4a).

Ante muy baja disponibilidad hídrica, ambos híbridos de Z.mays ejercieron un efecto similar sobre la tasa fotosintética de la maleza, diferenciándose $(p<0.05)$ del tratamiento control y de baja disponibilidad hídrica a los 6 y 15 días de comenzado el período de estudio respectivamente (Fig. 4a). La baja disponibilidad hídrica afectó significativamente la tasa fotosintética de Z.mays a los 6 días de iniciado el período de evaluación (Fig. 4b). Hacia el final del período de evaluación, la disminución en la tasa fotosintética se acentuó siendo de $75.7 \%$ para el tratamiento de baja disponibilidad hídrica. Bajo muy baja disponibilidad hídrica, la disminución de la fotosíntesis fue de $92.6 \%$ respecto al control $(p<0.01)$. Este tratamiento produjo una merma significativa $(p<0.05)$ en la tasa fotosintética ya a los 3 días de iniciado el período de evaluación (Fig. 4b). Para los dos tratamientos evaluados no hubo diferencias entre los híbridos de Z.mays.

Tal como se observó para la tasa fotosintética, S.halepense mostró una menor variación de la conductancia estomática a lo largo del período de evaluación respecto de aquella observada en ambos híbridos de Z.mays (Fig. 4c y Fig. 4d). La maleza mostró una disminución significativa $(p<0.05)$ a los 3 días de iniciado el período de estudio en el tratamiento de muy baja disponibilidad hídrica (Fig. 4c). En tanto, la baja disponibilidad hídrica, ejerció una disminución significativa $(p<0.05)$ a los 6 días de iniciado el período de evaluación.

La merma de la conductancia estomática en Z.mays mostró una tendencia similar a la registrada en la tasa fotosintética a lo largo del período de estudio. No obstante, ya al tercer día de establecida la competencia, el efecto de la muy baja disponibilidad hídrica ocasionó una caída altamente significativa $(p<0.01)$ de la conductancia estomática en ambos híbridos (Fig. 4d). A los 9 días de iniciado el período de evaluación, el híbrido Ax 840 presentó una mayor conductancia estomática que el híbrido Ax 888 ante una baja disponibilidad hídrica $(p<0.05)$. Esta diferencia que se mantuvo hasta los 18 días de iniciadas las evaluaciones. Este tratamiento produjo una merma de la conductancia estomática del $95 \%$, en tanto ante muy baja disponibilidad hídrica la disminución fue del $98 \%$ (promedio de ambos híbridos) (Fig. 4d). La tasa transpiratoria, tanto en S.halepense como en Z.mays, siguió una tendencia similar a la conductancia estomática (Fig. 4e y Fig. 4f).

\section{Complementariedad de recursos}

Excepto para la primera evaluación en el tratamiento de competencia en donde se registró un RYT superior a $1.0(p<0.05)$, en los dos años evaluados, ambos niveles de disponibilidad hídrica no registraron valores significativamente $(p<0.05)$ diferentes a 1.0, indicando la ausencia de complementariedad de recursos y la existencia de plena competencia entre el cultivo y la maleza (Tabla 1).

\section{Habilidad competitiva}

Ante una baja disponibilidad hídrica, S.halepense resulto ser más agresivo que ambos híbridos de Z.mays desde la primera semana de establecido el período de evaluación (Fig. 5). A partir del $9^{\text {no }}$ día de generado el período de competencia, S.halepense presentó una mayor agresividad compitiendo con el híbrido Ax 888 que aquella observada en competencia con el híbrido Ax 840. Esta diferencia de agresividad se mantuvo durante 9 días más, hasta el $18^{\text {vo }}$ día de iniciado el período de estudio (Fig. 5). La agresividad de S.halepense en el tratamiento de muy baja disponibilidad hídrica fue mayor aún que la registrada en el tratamiento de baja disponibilidad (Fig. 5). Esa mayor agresividad de la maleza ya se manifestó al $3^{\text {er }}$ día de iniciado el período de evaluación (Fig. 5).

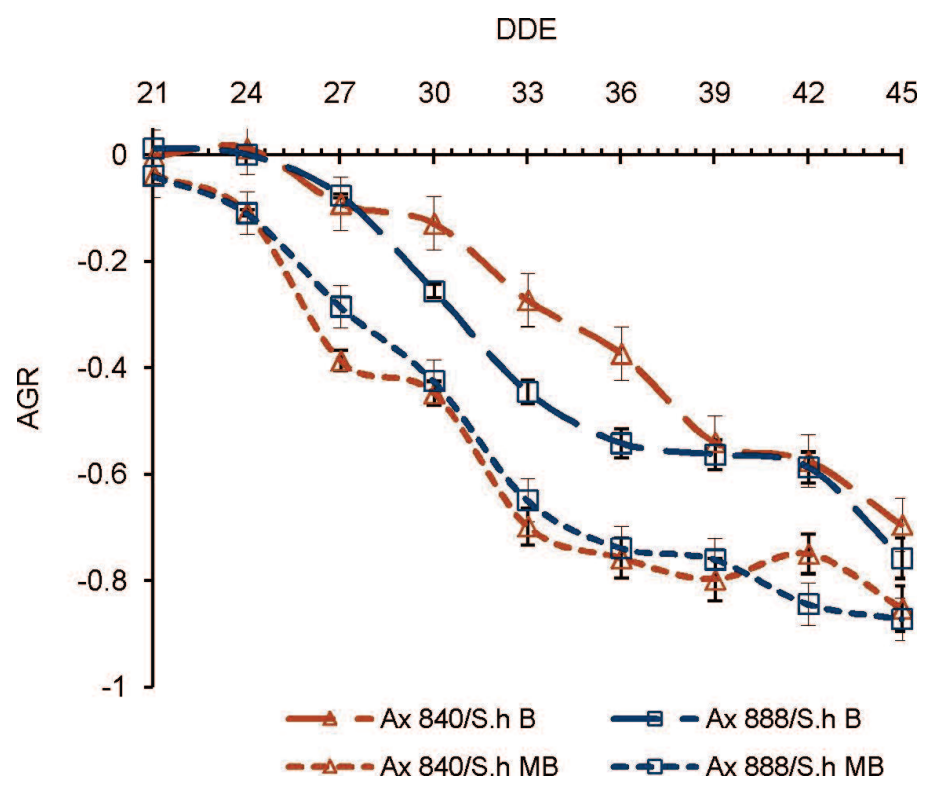

Figura 5. Habilidad competitiva medida a través del índice de agresividad $(A G R)$ con baja disponibilidad hídrica de Z.mays con S.halepense (Ax 840/S.h B y Ax 888/S.h B) y con muy baja disponibilidad hídrica (Ax 840/S. $h$ MB y Ax 888/S.h MB) a partir del establecimiento de los tratamientos 21 días desde la emergencia (DDE). Datos promedios para ambos años de estudio. La barra indica el error estándar (es). ( $n=5)$.

Tabla 1. Complementariedad de recursos medida a través del RYT en los tratamientos de baja disponibilidad hídrica de S.halepense con Z.mays (S.h/Ax 840 (B) y S.h/ Ax888 (B)) y de muy baja disponibilidad de S.halepense con Z.mays (S.h/Ax 840 (MB) y S.h/ Ax888 (MB)) a los 4, 7, 11, 16, 20 y 24 días de establecido el período de competencia. DMS: diferencia mínima significativa para cada evaluación (Tukey, $p<0,05)(n=5)$

RYT

Días de competencia

\begin{tabular}{|c|c|c|c|c|c|c|c|c|c|c|c|c|}
\hline & \multicolumn{6}{|c|}{$2002-2003$} & \multicolumn{6}{|c|}{ 2004-2005 } \\
\hline & 4 & 7 & 11 & 16 & 20 & 24 & 4 & 7 & 11 & 16 & 20 & 24 \\
\hline S.h/Ax 840 (B) & 1.11 & 1.05 & 1.06 & 1.06 & 1.05 & 1.04 & 1.12 & 1.06 & 1.06 & 1.05 & 1.04 & 1.04 \\
\hline S.h/Ax 888 (B) & 1.12 & 1.07 & 1.05 & 1.06 & 1.08 & 1.02 & 1.11 & 1.05 & 1.07 & 1.05 & 1.05 & 1.03 \\
\hline S.h/Ax 840 (MB) & 1.10 & 1.00 & 1.02 & 1.01 & 1.00 & 0.99 & 1.08 & 1.05 & 1.03 & 1.02 & 1.00 & 1.01 \\
\hline S.h/Ax 888 (MB) & 1.08 & 1.04 & 1.04 & 1.03 & 0.99 & 1.00 & 1.07 & 1.05 & 1.04 & 0.99 & 1.00 & 1.01 \\
\hline DMS $(p<0.05)$ & 1.10 & 0.08 & 0.07 & 0.07 & 0.10 & 0.09 & 0.09 & 0.07 & 0.08 & 0.06 & 0.06 & 0.05 \\
\hline
\end{tabular}




\section{Discusión}

\section{Complementariedad de Recursos}

En ambos años evaluados no hubo complementariedad de recursos entre las dos especies en los niveles de competencia ensayados, alcanzado una intensidad competitiva similar en ambos tratamientos. Como se estableció anteriormente estos valores de RYT son similares a los comunicados en estudios previos de competencia por recursos edáficos conducidos bajo riego periódico (Semere y Froud-Williams, 2001; Acciaresi et al., 2003). Es importante destacar que, aún en el tratamiento de baja disponibilidad hídrica (con una fluctuación del $\psi_{\mathrm{s}}$ entre -0.04 MPa a -0.2/-0.35 MPa en el intervalo de medición de tres días), se observa un considerable efecto competitivo entre ambas especies durante el período crítico de competencia de Z.mays.

\section{Evolución del Potencial Hídrico del Suelo $\left(\psi_{\mathrm{s}}\right)$, Potencial Hí- drico Foliar $\left(\psi_{\mathrm{f}}\right)$ y Contenido Relativo de agua (CRA)}

La competencia intensa del tratamiento de muy baja disponibilidad de agua produjo una disminución importante en los valores del $\psi_{\mathrm{s}}$, indicando que a lo largo del período de competencia evaluado hubo una extracción efectiva de agua por parte de las especies estudiadas. Esta merma fue mayor que la producida por la baja competencia, con fluctuación del $\psi_{\mathrm{s}}$. Comparando la dinámica del $\psi_{\mathrm{s}}$ y el $\psi_{\mathrm{f}}$ de cada uno de los genotipos evaluados, es posible que la merma en el $\psi_{\mathrm{s}}$ sea debida a la extracción que realizó $S$.halepense durante el período de competencia. Esto se fundamenta en que aún restituyendo el valor del $\psi_{\mathrm{s}}$ a valores cercanos a -0.04 MPa por medio del riego, el $\psi_{\mathrm{f}}$ de ambos híbridos de Z.mays no registró variación alguna. Davis et al. (1965) establecen que aquellas especies adaptadas a condiciones de baja humedad son las que alcanzan las mayores tasas de extracción hídrica en condiciones de competencia por agua en situaciones de deficiencia hídrica.

Es importante destacar que la muy baja disponibilidad hídrica rápidamente produjo una disminución del $\psi_{\mathrm{s}}$ a valores de $-1.0 \mathrm{MPa}$, situación que se correspondió con un 15-20\% de humedad en el suelo, visualizando en ambos híbridos de Z.mays, importantes síntomas de deficiencia de agua (senescencia foliar anticipada). En S.halepense, en cambio, no se observaron estos síntomas a lo largo del período de estudio. Este comportamiento quedó reflejado en el valor del CRA de la maleza, variable que realiza una estimación adecuada del estado hídrico celular bajo el posible efecto del $y_{s}$ y el ajuste osmótico.

En comparación con la merma del $\psi_{\mathrm{f}}$, el CRA, la conductancia estomática y la tasa transpiratoria disminuyeron relativamente menos en las plantas de S.halepense sometidas a competencia intensa por la muy baja disponibilidad hídrica. El mantenimiento de un CRA relativamente elevado a muy bajos $\psi_{\mathrm{f}}$ puede estar relacionado con una disminución del potencial osmótico $\left(\psi_{\text {os }}\right)$ y consiguiente mantenimiento del potencial de turgencia $\left(\psi_{\mathrm{f}}\right)$ en las hojas de la maleza. Esto podría ser debido a una mayor acumulación de osmolitos y/o interacción con proteínas (Smirnoff y Cumbes, 1989), que contribuiría a una mayor osmoprotección (Rhodes y Hanson, 1993) de la maleza en situación de competencia intensa. La tendencia observada en el $\psi_{\mathrm{f}}$ y en el CRA de S.halepense coincide con lo establecido por Stuart et al. (1985). Así, la rápida caída observada en el $\psi_{\mathrm{s}}$ en los tratamientos de competencia podría haberse debido a una mayor capacidad de ajuste osmótico de S.halepense respecto a la de ambos híbridos de Z.mays. Esto le habría permitido a la maleza mantener el crecimiento radical, favoreciendo la captación de agua a niveles decrecientes de $\psi_{\mathrm{s}}$ como determinaron Acciaresi y Guiamet (2010)

En los híbridos de Z.mays, se observó en la primera semana de competencia una estabilización del CRA en valores cercanos al $70 \%$, coincidiendo con valores de $\psi_{\mathrm{f}}$ de $-1.0 \mathrm{MPa}$, a pesar de restituirse cada tres días los valores de $\psi_{\mathrm{s}}$ a capacidad de campo. Este comportamiento está indicando una rápida pérdida de turgencia por parte del cultivo que pudo haber interferido con la capacidad de los híbridos de Z.mays para llevar adelante un A.O. y mantener el intercambio gaseoso (Bergonci et al. 2000). La estabilidad del $\psi_{\mathrm{f}} \mathrm{de}$ Z.mays en un valor cercano a -1.5 Mpa, se reflejó en una disminución de la turgencia (CRA) y en la disminución del intercambio gaseoso.

\section{Intercambio gaseoso}

Los cambios en el $\psi_{\mathrm{f}}$ y el CRA guardaron una estrecha relación con la dinámica del intercambio gaseoso. Los resultados obtenidos durante el período de competencia mostraron que S.halepense mantuvo un intercambio gaseoso (transpiración y fotosíntesis) de mayor intensidad que en los híbridos de Z.mays. Las tendencias encontradas en la tasa transpiratoria para los dos niveles de competencia estudiados reflejaron la habilidad de cada especie para captar el recurso por el cual compitieron. Acciaresi y Guiamet (2010) encontraron que $S$. halepense puede mantener la tasa de crecimiento relativa debido a una mayor longitud de raíces muy finas $(<240 \mu \mathrm{m})$ y la densidad de longitud de raíces, cuando crece en competencia por agua con Z.mays, alcanzando una mayor agresividad que el cultivo en dicha situación competitiva.

De acuerdo a los resultados alcanzados, fue posible observar que a medida que transcurría el período de competencia, la disminución de la conductancia estomática precedió a la merma alcanzada en la tasa fotosintética. Esto coincide con lo establecido por Chaves (1991) y Cornic (2000), quienes determinaron que la inhibición de la fotosíntesis en condiciones de déficit hídrico moderado es debida fundamentalmente a una restricción en la difusión de $\mathrm{CO}_{2}$. Sin embargo, Lawlor (2002) estableció que a medida que decrece el CRA, la tasa fotosintética disminuye inicialmente debido a una caída en la conductancia estomática y luego debido a causas metabólicas (no estomáticas), ocasionada fundamentalmente por una disminución en la síntesis de ribulosa 1-5 bifosfato (RUBP). No obstante, Flexas et al. (1998) y Maroco et al. (2002), determinaron que en condiciones de déficit hídrico prolongadas (días a semanas), luego de generarse una merma en la tasa fotosintética por la disminución de la conductancia estomática se produciría un nuevo ajuste de esta variable a fin de modular el nivel de actividad a la concentración intercelular de $\mathrm{CO}_{2}$ existente y al bajo nivel de crecimiento alcanzado. De este modo, dadas las tendencias visualizadas en ambas especies, es posible que en S.halepense la disminución en la tasa fotosintética haya sido debida fundamentalmente a causas estomáticas, en tanto la inhibición de la tasa fotosintética de los genotipos de Z.mays podría haberse debido a ambos tipos de limitaciones.

Asimismo, el comportamiento gaseoso visualizado en Z.mays puede ser considerado como una estrategia conservadora del agua edáfica tal como fuera definida por Ray et al. (1997), con un cierre estomático anticipado. De este modo, híbridos de Z.mays que muestren una estrategia de conservación del agua edáfica durante el período crítico de competencia $\mathrm{V}_{4}-\mathrm{V}_{7}$, podrían brindarle una ventaja competitiva a las malezas si estas presentan un "consumo de lujo" durante el período de competencia (Patterson, 1995). La mayor habilidad competitiva observada en S.halepense concuerda con lo establecido por Taiz y Zeiger (2006) y Vamerali et al. (2003) quienes determinan que un activo intercambio gaseoso ante la ocurrencia de períodos fluctuantes de disponibilidad de agua, tal como sucede con frecuencia durante el ciclo de los cultivos, permite alcanzar mayores tasas de crecimiento y el mantenimiento de la productividad, una vez restablecidos los niveles hídricos adecuados. De este modo, un activo intercambio gaseoso como el que presentó S.halepense, favorecería a la mayor agresividad (tanto en la captación de agua como en la producción de materia seca aérea) y afectaría la productividad del cultivo con el que compite. Radosevich et al. (1997) establecieron que la habilidad de una maleza para disminuir rápidamente la disponibilidad de agua edáfica y la capacidad de mantener el crecimiento bajo esas condiciones, es un mecanismo adecuado en situaciones de competencia por agua con los cultivos. 
El mantenimiento del intercambio gaseoso a bajos valores de $\mathrm{y}_{\mathrm{S}}$ alcanzado por S.halepense como así también el comportamiento estomático registrado, coincide con lo establecido por Patterson (1995) como respuesta general de ciertas especies de malezas consideradas "consumidoras" de humedad en situaciones de competencia. Estos estudios determinaron que el cultivo realizaba un ahorro de agua en competencia, en tanto las malezas presentaban un importante uso de agua. Así, mientras el cultivo tendía a optimizar el uso del agua a través del cierre estomático, las malezas continuaban absorbiendo dicho recurso a expensas del crecimiento del cultivo.

En el presente trabajo, S.halepense estableció una ventaja competitiva en ambos niveles de competencia ante híbridos de Z.mays con un comportamiento "conservador" de la humedad, indicando que la maleza podría mantener su agresividad en diferentes situaciones productivas en las que exista competencia por agua. En Argentina, la producción de maíz se lleva a cabo, mayoritariamente, bajo el sistema de secano. En tanto, el rendimiento del cultivo está principalmente condicionado por el régimen pluvial, el cual se caracteriza por un déficit hídrico de hasta tres semanas. En estas condiciones, la estrategia de consumo sostenido de agua hallada en S.halepense sería un factor relevante en la producción de maíz donde la disponibilidad hídrica condiciona la agresividad de la maleza. Sin embargo, no todas las especies de malezas presentes en los agroecosistemas presentarían la misma estrategia de captación de agua, por esta razón, es necesario seguir avanzando en la comprensión de esos fundamentos bioecológicos de la asociación cultivo-maleza para poder brindar nuevas estrategias de manejo.

\section{Conclusiones}

S.halepense mantuvo una absorción continua de agua a lo largo del período de competencia estudiado, independientemente del híbrido de Z.mays con el cual compitió.

Durante el período de competencia hubo una mayor disminución en el potencial hídrico foliar en S.halepense que en ambos híbridos de Z.mays, con un mantenimiento relativo de la turgencia foliar en la maleza, comportamiento posiblemente mediado por una mayor capacidad de ajuste osmótico. Este mantenimiento relativo de la turgencia (CRA) en S.halepense habría permitido mantener un activo intercambio gaseoso y podría alcanzar una mayor agresividad en la tasa transpiratoria durante el proceso competitivo por agua edáfica.

Los híbridos simples de Z.mays estudiados mostraron una estrategia de conservación de la humedad edáfica para los dos niveles de competencia evaluados, comportamiento que favoreció a S.halepense a través del consumo sostenido de agua que hizo esta especie.

El mantenimiento del intercambio gaseoso alcanzado en S.halepense otorga una mayor habilidad competitiva bajo los diferentes niveles de competencia hídrica evaluados frente a los dos híbridos de Z.mays.

S.halepense produjo una importante merma en la disponibilidad edáfica a la vez que pudo continuar con el intercambio gaseoso en dichas condiciones, características que ponen de manifiesto la agresividad que esta especie tendría en los sistemas agrícolas extensivos.

\section{Referencias}

Acciaresi, H.A., Chidichimo, H.O., Sarandón, S.J. 2003. Shoot and root competition in a Lolium multiflorum-wheat association. Biological Agriculture and Horticulture 21:15-33.

Acciaresi, H.A., Guiamet, J.J. 2010. Below- and above-ground growth and biomass allocation in maize and Sorghum halepense in response to soil water competition. Weed Research 50(5):481-492.

Bergonci, J.I., Bergamaschi, H., Berlato, M.A., Santos, O.S. 2000. Potencial da água na folha como um indicador de déficit hídrico em milho. Pesquisa Agropecuária Brasileira 35:1531-1540.

Bohnert, H.J., Bressan, R.A. 2001. Abiotic stresses, plant reactions, and approaches towards improving stress tolerance. En: Nösberger, J., Geiger H.H., Struik, P.C. (eds.), Crop Science: Progress and prospects, pp. $81-$ 100. CABI Publishing, Wallingford. UK

Chaves, M.M. 1991. Effects of water deficits on carbon assimilation. Journal of Experimental Botany 42:1-16.

Cornic, G. 2000. Drought stress inhibits photosynthesis by decreasing stomatal aperture not by affecting ATP synthesis. Trends in Plant Science 5:187-188.

Cornic, G., Fresneau, C. 2002. Photosynthetic carbon reduction and carbon oxidation cycles are the main electron sinks for Photosystem II activity during a mild drought. Annals of Botany 89:887-894.

Flexas, J., Escalona, J.M., Medrano, H. 1998. Down-regulation of photosynthesis by drought under field conditions in grapevine leaves. Australian Journal of Plant Physiology 25:893-900.

Lawlor, D.W. 2002. Limitation to photosynthesis in water-stressed leaves: stomata vs. metabolism and the role of ATP. Annals of Botany 89 (special number): $871-885$

Patterson, D.T. 1995. Effects of environmental stress on weed/crop interactions. Weed Science 43:483-490.

Maroco, J.P., Rodrigues, M.L., lopes, C., Chaves, M.M. 2002. Limitations to leaf photosynthesis in grapevine under drought - metabolic and modeling approaches. Functional Plant Biology 29:1-9.

Radosevich, S., Holt, J., Ghersa, C.M. 1997. Other types of interference. En: Radosevich, C., Holt, J., Ghersa, C.M. (eds.) Weed ecology. Implications for management, pp. 302-311. John Wiley y Sons, Inc. NY, USA.

Rajcan, I., Swanton, C.J. 2001. Understanding maize-weed competition: resource competition, light quality and the whole plant. Field Crops Research 71:139-150.

Ray, J.D., Samson, B.K., Sinclair, T.R. 1997. Vegetative growth and soil water extraction of two maize hybrids during water deficits. Field Crops Research 52:135-142.

Rhodes, D., Hanson, A.D. 1993. Quaternary ammonium and tertiary sulfonium compounds in higher plants. Annual Review of Plant Physiology and Plant Molecular Biology 44:357-384.

Semere, T., Froud-Williams, R.J. 2001. The effect of pea cultivar and water stress on root and shoot competition between vegetative plants of maize and pea. Journal of Applied Ecology 38:137-145.

Smirnoff, N., Cumbes, Q.J. 1989. Hydroxyl radical scavenging activity of compatible solutes. Phytochemistry 28:1057-1060.

Stuart, B.L., Krieg, D.R., Abernathy, J.R. 1985. Photosynthesis and stomatal-conductance responses of Johnsongrass (Sorghum halepense) to water stress. Weed Science 33:635-639.

Taiz, L., Zeiger, E. 2006. Water Balance of Plants. En: Taiz, L., Zeiger E.(eds.). Plant Physiology. Sinauer Associates, Inc. Publishers. Sunderland. Disponible en: http://4e.plantphys.net/categories.php?t=t.

Thomas, P., Allison, J. 1975. Competition between maize and Rottboellia exaltata. Journal of Agricultural Science 84:305-312.

Turner, N.C. 2001. Optimising water use. En: J Nösberger, HH Geiger, PC Struik (eds), pp: 119-135. Crop science: progress and prospects. CABI Publishing, Wallingford, UK.

Vamerali, T., Saccomani, M., Bona, S., Mosca, G., Guarise, M., Ganis, A. 2003. A comparison of root characteristics in relation to nutrient and water stress in two maize hybrids. Plant and Soil 255:157-167. 